\title{
Investigation of Removal Capacities of Biofilters for Airborne Viable Micro-Organisms
}

\author{
Rémi Soret ${ }^{1}$, Jean-Louis Fanlo ${ }^{1}$, Luc Malhautier ${ }^{1}$, Philippe Geiger ${ }^{2}$ and Sandrine Bayle ${ }^{1, *}$ \\ 1 LGEI, IMT Mines Ales, University of Montpellier, 30100 Ales, France; remi.soret@mines-ales.fr (R.S.); \\ jean-louis.fanlo@mines-ales.fr (J.-L.F.); luc.malhautier@mines-ales.fr (L.M.) \\ 2 CMI Europe-Environnement, 1 rue des Pins, Parc d'Activités du Pays de Thann, 68700 Aspach-Michelbach, \\ France; philippe.geiger@cmigroupe.com \\ * Correspondence: sandrine.bayle@mines-ales.fr; Tel.: +33-046-678-2708
}

Received: 24 January 2018; Accepted: 1 March 2018; Published: 19 March 2018

\begin{abstract}
New emerging issues appears regarding the possible aerosolization of micro-organisms from biofilters to the ambient air. Traditional bioaerosol sampling and cultural methods used in literature offer relative efficiencies. In this study, a new method revolving around a particle counter capable of detecting total and viable particles in real time was used. This counter (BioTrak 9510-BD) uses laser-induced fluorescence (LIF) technology to determine the biological nature of the particle. The concentration of viable particles was measured on two semi-industrial pilot scale biofilters in order to estimate the Removal Efficiency in viable particles $\left(R E_{v p}\right)$ in stable conditions and to examine the influence of pollutant feeding and relative humidification of the gaseous effluent on the $\mathrm{RE}_{\mathrm{vp}}$. The $\mathrm{RE}_{\mathrm{vp}}$ of biofilters reached near $80 \%$ and highlighted both the stability of that removal and the statistical equivalence between two identical biofilters. Pollutant deprivation periods of $12 \mathrm{~h}, 48 \mathrm{~h}$ and 30 days were shown to have no influence on the biofilters' removal capacity, demonstrating the robustness and adaptation capacities of the flora. In contrast, a 90-day famine period turned the biofilters into emitters of viable particles. Finally, the humidification of the effluent was shown to negatively influence the removal capacity for viable particles, as drying off the air was shown to increase the $\mathrm{RE}_{\mathrm{vp}}$ from 60 to $85 \%$.
\end{abstract}

Keywords: biofiltration; bioaerosols; BioTrak; fluorescence; operating parameters

\section{Introduction}

Air pollution control has been a concern for many years. To preserve air quality and limit emissions of chemical pollutants, several laws have been established at national, European and international scales. For example, the regulations toward air pollution control tightened over the last years for Volatile Organic Compounds (VOCs) and odors (Directive 2008/50/EC). To comply with those regulations, biological processes such as biofilters have been implemented for the treatment of gaseous effluents in a wide range of environments like manufacturing industries (paper industries, chemical industries, etc.), wastewater treatment plants, biogas production units (methanization) or composting plants [1]. Biofilters are one of the best available techniques [1,2]. They are appropriate for treating gaseous stream with high flow rate at low concentration of pollutants. They offer a sustainable and eco-friendly solution for air pollution control, with low maintenance costs. For all those reasons, their implementation at industrial scale grew exponentially in recent decades. However, as biofilters are biological systems themselves, further focus has been set on the question of if they act as additional sources of airborne microorganisms. Hence, the dispersion of microorganisms from gas biofilters and their health consequences for workers and residents are the subject of discussions. During the biofiltration process, gaseous effluent is forced through a packing bed material colonized by a microbial flora which enables the degradation of the pollutant [3]. 
This biological mechanism leads to interrogations about the possible aerosolization of micro-organisms from the biofilm to the gaseous effluent and their diffusion in the atmosphere [4-6]. Some studies demonstrate adverse health effects from occupational exposure to bioaerosols produced during biological process functioning [7]. These questions have led to the publication of Directive 2000/54/EC by the European Parliament on the protection of workers from risks related to exposure to biological agents at work. The British Government recently emitted a new regulation enforcing the monitoring of bioaerosols in biological waste treatment plants [8]. In ambient air, the World Health Organization (WHO) indicated that the exposure limit value (ELV) of bioaerosol in ambient air should be lower than $300 \mathrm{CFU} \cdot \mathrm{m}^{-3}$ [9].

Moreover, the inlet gaseous influent can contain a large amount of micro-organisms or some pathogenic micro-organisms according to the industrial activity (rendering, biogas production ... ). A few studies investigated those questions by using generally classical microbiological methods [6]. However, it is well known that these methods are rather laborious (depending on the culturability of microorganisms) and time consuming (from 2 to 10 days) [10]. Moreover, Amann et al. [11] reported that the percentage of cultivable micro-organisms in an environment represent only from 0.01 to $10 \%$ of the total microbial flora. Cultivable cells only represent 4 to $30 \%$ of the total biomass in gas biofilters [12,13]. Furthermore, the collection methods and growth media used to study emissions of micro-organisms from gas biofilters were variable.

The profusion of approaches has made it difficult to compare results $[6,9,14-20]$. To overcome these problems, new approaches called rapid microbiological methods (RMM) appeared. Among them, methods to determine the number of microorganisms via evaluation of microbial viability have been developed [21]. One of those emerging techniques is based on laser/light-induced fluorescence

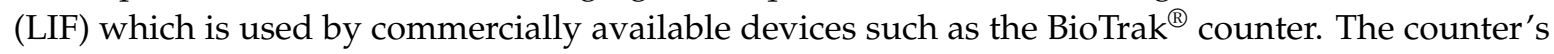
utilization has been validated by the USFDA [22] and its performances as an alternative method of microbiology have been validated in accordance with USP Validation of Alternative Microbial Methods (USP <1223> [23], USP <1116> [24]), the European Pharmacopoeia (Ph. Eur. Chapter 5.1.6 [25]) and the Parenteral Drug Association (PDA Technical Report 33 [26]). This device allows an easy and fast monitoring of total viable airborne flora with a cut-off size of $2 \mu \mathrm{m}$ [27], which is relevant to the 2-10 $\mu \mathrm{m}$ aerodynamic diameter range where the majority of bioaerosol can be found [28]. In their review, Fletcher et al. [6] highlighted the need for regular monitoring of bioaerosol emissions from biofilters during steady state operation by using a standardized and repeatable measurement method. Moreover, it is also of great importance to examine the influence of operating parameters on the potential emission of microorganisms.

They also emphasized the use of a parameters controlled biofiltration pilot unit to eliminate the high variability of industrial field sampling. The BioTrak counter could be an efficient tool for that purpose. The first goal of this study is then to determine the $\mathrm{RE}_{\mathrm{vp}}$ of a biofiltration pilot unit at steady state by monitoring its emissions of viable particles. The second goal is to investigate the influence of operating parameters on the $\mathrm{RE}_{\mathrm{vp}}$ via controlled perturbations. The influence of two operating parameters such as pollutant feeding and humidity of the effluent has been presented here.

\section{Materials and Methods}

\subsection{Optical Total and Viable Particles Counter BioTrak ${ }^{\circledR}$}

The main device used in this study is the Optical Total and Viable Particles Counter BioTrak ${ }^{\circledR}$ (BioTrak 9510-BD, TSI, Shoreview, MN, USA). The counter is composed of two distinct stages. The first one hosts an Optical Particles Counter (OPC). This counter uses laser diffraction to measure and count every solid particle, including inorganic and organic particles, in the diameter range from 0.5 to $25 \mu \mathrm{m}$. Among these particles, viable particles are then determined by auto fluorescent detection.

For this detection, the particles go through a cyclonic concentrator which reduces the airflow while maintaining the majority of the particles inside, to then reach the second stage. 
This corresponds to the viability detector which uses laser-induced fluorescence to discriminate the viable particles in the total exiting the concentrator. For this, two $405 \mathrm{~nm}$ wave length lasers enable the fluorescence of the NADH, Flavin and tryptophan molecules associated with viability.

This device allows the real-time monitoring of the viable airborne flora. The sampling flowrate is 28.3 $\mathrm{L} \cdot \mathrm{min}^{-1}$. Measurements using the BioTrak counter consisted of 15 cycles: a measurement of $1 \mathrm{~min}$ followed by a pause of $30 \mathrm{~s}$.

\subsection{Pilot Scale Biofiltration Unit}

The unit is composed of 2 major parts: the gaseous effluent generation and the two biofiltration units in parallel (Figure 1).

A synthetic gaseous effluent containing a carbon concentration of around $7.5 \mathrm{~g} \cdot \mathrm{carbon} \cdot \mathrm{m}^{-3}$ air at a flow rate of $20 \mathrm{~L} \cdot \mathrm{min}^{-1}$ was created by injecting SP95 petrol into compressed air stream prior to reaching the carrier effluent. The carrier effluent is composed of ambient air aspired by a centrifugal air fan and going through a humidification tower to reach maximum water saturation (95\%) before being distributed among the biofilters, in an upward flow mode, at a flow rate of $2 \mathrm{~m}^{-3} \cdot$ air $\cdot \mathrm{h}^{-1}$ and an inlet VOCs concentration of $1 \mathrm{~g} \cdot$ carbon $\cdot \mathrm{m}^{-3}$. Biofiltration columns are identical PVC columns of $3 \mathrm{~m}$ in height and $30 \mathrm{~cm}$ in diameter and packed with sawmilled wooden chips with an average size of $10 \mathrm{~mm}$ up to $1 \mathrm{~m}$ height. Both were operated in parallel during all experiments. A $50 \mathrm{~cm}$ head space at the top of each column contained the humidification system. A $55 \mathrm{~cm}$ bottom section is designed for gas velocity homogenization prior to entering the packing bed, as well as for leachate recovery. In order to maintain the humidity of the packing bed material, the biofilters were watered with standard water at a flow of $15 \mathrm{~L} \cdot \mathrm{m}^{-2} \cdot$ day $^{-1}$.

The biofilters were inoculated by placing the packing bed material inside sewage sludge coming from the local wastewater treatment plant for $24 \mathrm{~h}$. Ambient temperature was controlled in the laboratory test hall assuring a minimum temperature of $15^{\circ} \mathrm{C}$. The inlet stream load was of $360 \mathrm{~g}$ of pollutant per cubic meter of packing bed per day ${ }^{-1}\left(360 \mathrm{~g} \cdot \mathrm{m}^{-3} \cdot\right.$ day $\left.^{-1}\right)$.

For this study, the supply of pollutant followed an alternation of $12 \mathrm{~h}$ of feeding followed by $12 \mathrm{~h}$ of deprivency for 5 days (Monday to Friday), then a period of complete deprivation for 2 days (Saturday and Sunday). This set up was chosen to represent typical full-scale biofilters operation in the oil industry (refining, storage, treatment of petroleum wastes). The average pollutant removal efficiency during this study was of $30 \%$.

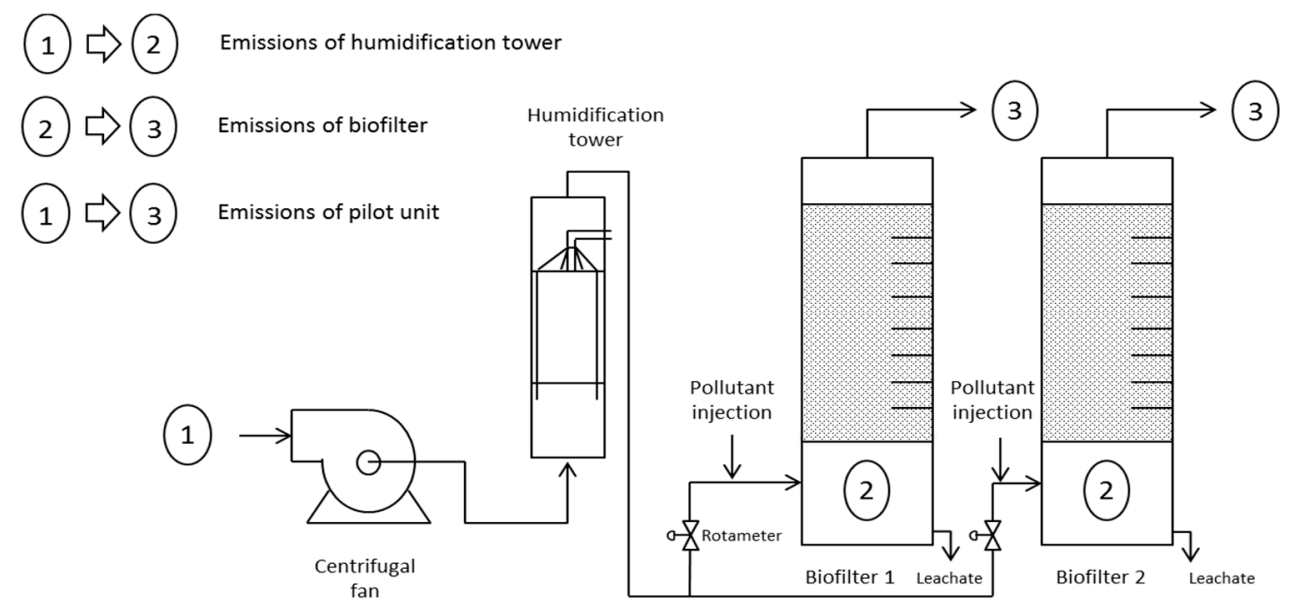

Figure 1. Schema diagram of experimental design with particles sampling points. 1: Ambient air. 2: Gas homogenization area. 3: Gas outlet. 


\subsection{Experimental Design}

\subsubsection{Sampling Points}

A schematic representation of the sampling points used is presented in Figure 1.

The first sampling point corresponded to the ambient air. It was placed approximately at $1.5 \mathrm{~m}$ from the floor and $1.5 \mathrm{~m}$ from the fan aspiration to ensure isolation and representativeness of the samplings. The second sampling location was inside the gas homogenization area and was sampled through the leachate outlet.

The third sampling point corresponds to the air exiting the biofilter, and was sampled directly inside the head space at the top of the column within a space located at $10 \mathrm{~cm}$ from the packing material. The $\mathrm{RE}_{\mathrm{vp}}$ of the pilot unit as a whole, of the humidification tower, and of the biofilter could be estimated by comparing the sampling results of points 1 and 3; points 1 and 2; points 2 and 3 respectively.

The results are expressed as removal efficiency of viable particles $\left(R E_{v p}\right)$. The $R E_{v p}$ is calculated as follows:

$$
\operatorname{REvp}(\%)=\left(\frac{C \text { input }- \text { C output }}{C \text { input }}\right) \times 100
$$

where $\mathrm{C}$ input corresponds to ambient air concentration $\left(\mathrm{VP} \cdot \mathrm{m}^{-3}\right)$ and $\mathrm{C}$ output to outlet gas concentration $\left(\mathrm{VP} \cdot \mathrm{m}^{-3}\right)$.

\subsection{2. $\mathrm{RE}_{\mathrm{vp}}$ at Steady State}

The emissions of viable particles of biofilters at steady state were investigated and the $R E_{\mathrm{vp}}$ determined during 44 days for both columns operating with strictly identical conditions. Two measurements per day were conducted to take into account both daily variations and pollutant feeding alternation. The first measurement was performed in the morning and the second one in the afternoon.

\subsubsection{Pollutant Feeding}

The influence of pollutant feeding was studied by depriving the biofilters of any pollutant for controlled periods of time while maintaining the same airflow. The first starvation period represented the alternation of pollutant feeding at steady state, thus deprivation periods of 12 and $48 \mathrm{~h}$. The second starvation period was of 90 days and started after a 3 months period of steady state. Following this starvation period, the biofilters were put back in steady state operations for 15 days. The final starvation period took place afterward and lasted 30 days. During feast conditions, both biofilters were fed with basal VOCs concentration $\left(360 \mathrm{mg} \cdot \mathrm{m}^{-3}\right)$. Each famine period was only applied when all biofilters experienced steady state.

\subsubsection{Gas Humidification/Moistening}

The influence of the air moisture content was examined by shutting down the humidification tower of the pilot unit for up to $50 \mathrm{~h}$. No further time was added in order to preserve the biofilm on the packing bed material.

A complementary analysis of the quantity of water in the air was performed during this period. Both air temperature and relative humidity were determined by using a temperature and hygrometry probe (KIMO instruments, Montpon Ménestérol, France), and the quantity of airborne water was computed with data from a Mollier diagram. Measurements were carried out in the ambient air, at the bottom and at the top of the biofilter to estimate the emitted quantity of water for each section of the experimental design by using a water mass balance. 
The determination of viable particle quantities in the air using the BioTrak counter was simultaneously performed, thus allowing the specific isolation of the share of the biofilter itself in the removal efficiency of the total pilot unit.

\subsection{Statistical Analysis}

Different statistical tests were used during this study. Analysis of variance was used in priority. For non-normally distributed variables, data were compared with Wilcoxon test. ANOVA was used to analyze steady state and humidification studies' data. Wilcoxon test was used for the pollutant deprivation study. The equivalence one-sided test (TOST) was used to determine the statistical equivalence between both biofilter columns. All analyses were performed using the statistic software R. The chosen level of risk was $5 \%$, a result was thus considered statistically significant with a $p$-value $<0.05$.

\section{Results and Discussion}

\subsection{Steady State}

Viable particles' concentrations (VP concentration) were monitored at steady state for a period of 44 days for both biofilters. The variation of the inlet VP concentration in the entire study (steady state, influence of pollutant and influence of humidity included) was limited to around $1 \mathrm{log}$ maximum, ranging from 900 to $12,000 \mathrm{VP} \cdot \mathrm{m}^{-3}$. The median inlet concentration was calculated as being $3200 \mathrm{VP} \cdot \mathrm{m}^{-3}$ and the mean concentration was $3800 \pm 2400 \mathrm{VP} \cdot \mathrm{m}^{-3}$ which highlighted the stability of the concentration throughout the study. The outlet VP concentration during the steady state study ranged from 270 to $3700 \mathrm{VP} \cdot \mathrm{m}^{-3}$ with a mean concentration of $950 \pm 750 \mathrm{VP} \cdot \mathrm{m}^{-3}$ for BF1. The concentration ranged from 20 to 2600 with a mean concentration of $970 \pm 750 \mathrm{VP} \cdot \mathrm{m}^{-3}$ for BF2. On average, the emissions were also lower than what reported in the literature [5,29-37]. However, the majority of these studies revolved around field sampling on on-site biofilters in highly contaminated environments. However, these results are higher than the WHO recommendation for bioaerosol concentration $\left(300 \mathrm{UFC} \cdot \mathrm{m}^{-3}\right)$ [9].

The small variation in VP concentrations in inlet effluent ensured the representativeness of the $\mathrm{RE}$ range variations. This parameter was therefore chosen to monitor changes in biofilter removal capacities of VP. $R E_{\mathrm{vp}}$ values are presented in Figure 2. The $\mathrm{RE}_{\mathrm{vp}}$ were of $79 \pm 17 \%$ and $76 \pm 19 \%$ for biofilter 1 (BF1) and biofilter 2 (BF2) respectively.

These values were in agreement with microbial RE values which have been reported by different authors for bacterial and fungal communities: from 30-98\% for bacteria [5,29-37], and from $49-100 \%$ for total fungi $[5,29-33,37]$. It is important to underline that these previous works were performed using on-site industrial biofilters. Hence, design characteristics, operating parameters and packing bed materials of these biofilters differed from one another. Furthermore, results were gathered using different sampling devices and sampling methods. The observed seemingly high variability among all these data is then due to both biofilter design and operation, and microbial particles sampling and counting. It should also to be noted that those results (except for [35]) were obtained through culturable methods with significant delays. It is also well known that these methods involve challenges for the representation of airborne microorganisms [10]. According to Amann et al. [11], these studies could have overlooked up to $90 \%$ of the total airborne microbial flora. The BioTrak technology has been chosen to overcome the cultivability of micro-organisms and to improve the microflora representation.

A few studies have implanted culture independent methods to observe airborne micro-organisms from biofilter. Epifluorescence Microscopy and FISH analysis have been used by Esquivel-Gonzalez et al., 2017 [9] and Ho et al., 2008 [35] respectively. Esquivel-Gonzalez et al., 2017 have found a weak emission from their biofilters (average inlet concentration: $3 \times 10^{7} \mathrm{cells} \cdot \mathrm{m}^{-3}$ air and average outlet concentration: $3.8 \times 10^{7} \mathrm{cells} \cdot \mathrm{m}^{-3}$.air). Using the FISH technique, $\mathrm{HO}$ et al., 2008 found a RE for total micro-organisms of $90 \%$ and $98 \%$ which is concordant with our results. 
Epifluorescence Microscopy include in their quantification non-viable cells or microbial fragments in the count (Esquivel-Gonzalez et al., 2017). Comparison of the target to be measured could explain the variation in the results. Culture methods highlight viable and cultivable micro-organisms population, BioTrak targets the viable micro-organisms, EM detects total cells (viable, dead and fragments) and FISH detects the DNA. There is a need to explore further the differences in measurement of different technologies.

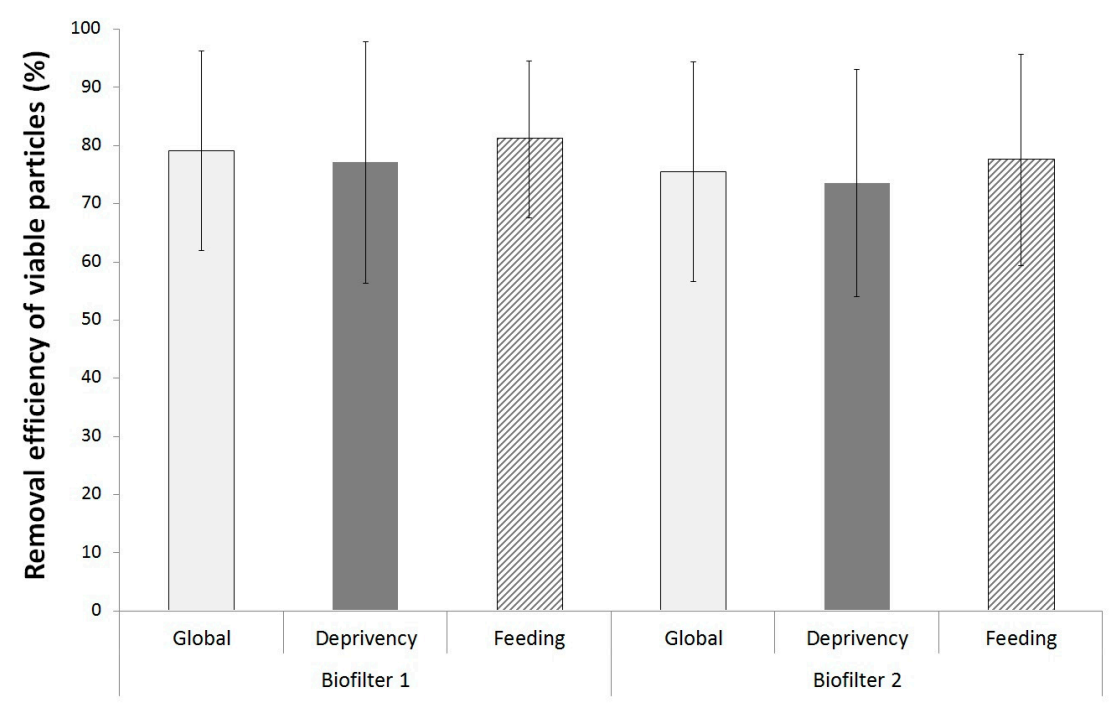

Figure 2. $\mathrm{RE}_{\mathrm{vp}}$ for both biofilters during the pollutant alternation of deprivency and feeding periods $\left(n_{\text {deprivency }}=25 /\right.$ bars, $n_{\text {feeding }}=27 /$ bars $)$.

Statistical analysis showed no difference between the feeding and deprivency periods $(p=0.3)$. Both 12 and $48 \mathrm{~h}$ of deprivency period included in the normal operation feeding scheme had no effect upon the $\mathrm{RE}_{\mathrm{vp}}$ levels emitted by biofilters (Figure 2). Moreover, no statistical difference has been detected on $\mathrm{RE}_{\mathrm{vp}}$ between both biofilters $(p>0.8)$.

The equivalence test demonstrated that both biofilters, identical in term of designs and operating conditions, presented equivalent removal efficiency for viable particles $(p<0.05)$.

\subsection{Pollutant Feeding}

Viable particles concentrations have been monitored to test the ecosystem resistance to a famine period. Both biofilters have been submitted to different famine periods ( $12 \mathrm{~h} / 48 \mathrm{~h}, 30$ days, and 90 days).

The $\mathrm{RE}_{\mathrm{vp}}$ have been calculated and summarized in Figure 3. In stable operating conditions (12 h/48 h famine periods) identical efficacy of $79 \pm 17 \%$ have been observed (Section 3.1). Two deprivation periods of 30 days have been performed, the mean outlet VP concentration was $960 \pm 700 \mathrm{VP} \cdot \mathrm{m}^{-3}$. The average $\mathrm{RE}_{\mathrm{vp}}$ was of $76 \pm 4 \%$.

During the 30 days period, the outlet concentration was remarkably similar to the steady state ones, showing no modification of the $\mathrm{RE}_{\mathrm{vp}}$. During the 90 days deprivation period, the mean inlet concentration was $2700 \pm 1500 \mathrm{VP} \cdot \mathrm{m}^{-3}$. The data showed a negative average removal efficiency of $-51 \pm 37 \%$ during this period. The outlet concentration was significantly higher and highlighted the microbial aerosolization. After the seven day recovery period, the average concentration went back to $1000 \pm 640 \mathrm{VP} \cdot \mathrm{m}^{-3}$ and ranged from 570 to $1500 \mathrm{VP} \cdot \mathrm{m}^{-3}$. The concentration after the seven day recovery period also underscored the resilience of the system as it went back to steady state equivalent level. 
None of the $12 \mathrm{~h}, 48 \mathrm{~h}$ and 30 days deprivation periods had an influence on the levels of the $\mathrm{RE}_{\mathrm{vp}}$ $(p<0.01)$. Biofilter turned into an emitter of viable particles with a negative average $\operatorname{RE}_{\mathrm{vp}}(p<0.001)$ after 90 days of pollutant deprivation. When both biofilters were re-supplied with pollutants, a single period of seven days was required to reverse the phenomenon back to steady state, with an average efficiency level similar to that obtained before the 90-day famine period.

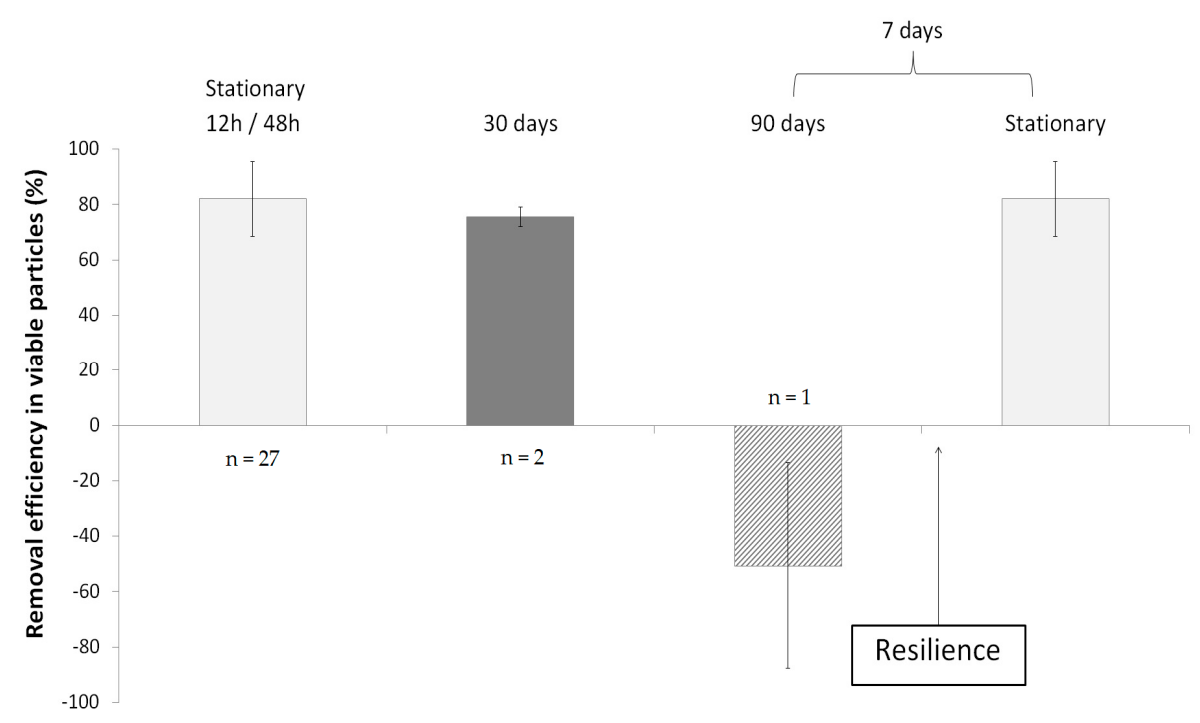

Figure 3. $R E_{v p}$ during the different deprivency periods ( $12 \mathrm{~h} / 48 \mathrm{~h}, 30$ and 90 days). The time required to recover the $\mathrm{RE}_{\mathrm{vp}}$ at steady state is of seven days.

Those results highlighted several phenomena.

The robustness of biofiltration toward short-term famine, (12-48 $\mathrm{h}$ and 30-day periods) could supposedly come from the ability of the microbial flora to use the substrate as a source of energy, which is an already reported phenomenon [38-42]. When the concentration of pollutant severely dropped during the deprivation period, the biofilter's microflora was able to slow down its metabolism and triggered a famine adaptation mechanism. It is probable that both carbon and energy sources were supplied by the packing material to compensate for the lack of pollutant.

Then, the microbial community organization was not disturbed for at least 30 days, as seen here, with no modification on the level of viable particles within the aerosol and the $\mathrm{RE}_{\mathrm{vp}}$.

However, after a longer famine period (90 days) microbial emission was observed, as around $50 \pm 37 \%$ more micro-organisms exited the biofilter than entered it. All $\mathrm{RE}_{\mathrm{vp}}$ values remained under $0 \%$ thus further highlighting the aerosolization of viable particles. This period seemed to be sufficient to overcome the adaptation mechanism of the microbial community. As the environmental conditions for microbial growth were unfavorable, the microflora developed a strategy of conservation (migration strategy) to find another suitable environment.

When both biofilters were re-supplied with a polluted gaseous effluent, a rapid return (one week) to the steady state $\mathrm{RE}_{\mathrm{vp}}$ was observed. The pollutant removal efficiency also took one week to return to pre-disturbance level (data not shown). This highlighted the resilience of biofilters toward long term famine. The possible explanation is that the renewed access to food stopped the migration survival mechanism of the microbial flora.

The results seemed to highlight the robustness of biofilters in terms of viable particles removal and that the biofilters and the associated microflora could survive long-term famine periods (at least 30 days). 


\subsection{Influence of the Effluent Humidification}

The measurements at the foot and the top of the biofilter allowed for the specific isolation of the biofilter column and showed that it possessed an average removal efficiency of $90 \pm 4 \%$. During the test period with the humidification tower working, the inlet concentration was of $2900 \pm 1500 \mathrm{VP} \cdot \mathrm{m}^{-3}$ $(n=6)$, while the inlet concentration was of $2400 \pm 400 \mathrm{VP} \cdot \mathrm{m}^{-3}(n=3)$ without humidification. The levels of the inlet concentrations were thus similar in both cases. As seen in the steady state study, this difference in the concentrations range could not explain the drastic difference between the $R E_{v p}$ with air humidification and the $R E_{v p}$ without humidification. The difference in the $R E_{v p}$ thus could not be imputed to a drastic difference of the inlet concentration but to the removal of air moisture. In the same way, the mean outlet VP concentration were of $1200 \pm 420 \mathrm{VP} \cdot \mathrm{m}^{-3}$ during humidification and $300 \pm 160 \mathrm{VP} \cdot \mathrm{m}^{-3}$ without, which highlighted the augmentation of the $\mathrm{RE}_{\mathrm{vp}}$ due to air moisture removal. In addition, these results demonstrate that the biofilter is the main part of the $R E_{v p}$, thus confirming the biofilter ability to remove viable particles.

The $\mathrm{RE}_{\mathrm{vp}}$ levels have been obtained with a pilot unit containing a humidification tower before the biofilter (Figure 1). To further investigate the particles retention of the biofilter itself, the determination of particle concentration between the tower and biofilter was attempted. However, the air humidity in this area prohibited the use of counter. Consequently, the humidification tower was stopped for up to $50 \mathrm{~h}$ which allowed to investigate of the biofilter $\mathrm{RE}_{\mathrm{vp}}$ itself.

When the humidification tower was working, the quantity of water delivered to the gas was estimated at $18 \mathrm{~g} \cdot \mathrm{h}^{-1}$ Table 1a. At the same time, the humidity content in the gas at both inlet and outlet of the column was similar which emphasized the stability of the moisture of the packing bed through time.

The quantity of water at the outlet of the biofilter itself has been determined after the humidification tower has been stopped (Table 1b). The obtained values seem to highlight the drying of the packing bed material (the quantity of water at the biofilter outlet was of $22.5 \mathrm{~g} \cdot \mathrm{h}^{-1}$ and of $15 \mathrm{~g} \cdot \mathrm{h}^{-1}$ at 22 and $46 \mathrm{~h}$ respectively).

Table 1. (a) Air temperature and relative humidity at the different localizations in the pilot unit, airborne water concentration calculated using Mollier diagram and airborne water flow corresponding to the airflow $\left(2 \mathrm{~m}^{3} \cdot \mathrm{h}^{-1}\right)$. (b) Quantity of water exiting the biofilter through time after the tower stoppage, calculated using airborne water mass balance between the bottom and the top of the biofilter.

\begin{tabular}{ccccc}
\hline (a) & $\mathbf{T}\left({ }^{\circ} \mathbf{C}\right)$ & HR (\%) & $\mathbf{C}_{\text {eau }}\left(\mathrm{g}_{\text {eau }} \cdot \mathbf{k g}_{\text {air }}{ }^{-\mathbf{1}}\right)$ & $\mathbf{Q}_{\text {eau }}\left(\mathrm{g} \cdot \mathbf{h}^{-\mathbf{1}}\right)$ \\
\hline Ambiant air & 20 & 55 & 8 & 19 \\
Tower exit & 21.5 & 95 & 15 & 37 \\
Biofilter exit & 21.5 & 95 & 15 & 37 \\
\hline (b) & Time & Biofilter & \\
\hline & $3 \mathrm{~h}$ & $22 \mathrm{~g} \cdot \mathrm{h}^{-1}$ & \\
& $22 \mathrm{~h}$ & $23 \mathrm{~g} \cdot \mathrm{h}^{-1}$ & \\
& $46 \mathrm{~h}$ & $15 \mathrm{~g} \cdot \mathrm{h}^{-1}$ & \\
\hline
\end{tabular}

The levels of $R E_{v p}$ of the pilot unit with or without humidification as well as the $R E_{v p}$ of biofilter itself are presented in Figure 4 . Those results exhibited an average $\mathrm{RE}_{\mathrm{vp}}$ of $60 \pm 9 \%$ during normal operation with humidification for the pilot unit in totality. After $46 \mathrm{~h}$ without air moistening, the average $R E_{v p}$ increased to an average of $86 \pm 9 \%$ which is statistically different to when the gaseous effluent is moistened $(p<0.03)$.

When the packing bed material dried, the $\mathrm{RE}_{\mathrm{vp}}$ increased drastically. The absence of water saturation of the packing bed seemed to decrease the crossing of the biofilter by the microbial flora, or its aerosolization. 
Vergara-Fernandez et al., 2012 [43] have reported an opposite result: a decrease of fungal spores' concentration concomitant with an increase of packing material. This opposite result could be explained by several factors. Actually, the scale up effect of the biofilter ( $1000 \mathrm{~L}$ for us against $0.5 \mathrm{~L}$ ) must be taken into account, as the scale-up effects inherent to a $2000 \%$ augmentation in volume can drastically change the results. Biofilter used in this study is packed with a consortium while a mono specie biofilter was used by Vergara-Fernandez et al., 2012, which directly affected the diversity of the bioaerosols emitted. Sporulation effects had a major influence on the results. The humidity increase may have altered the sporulation. Finally, the method of detection could have influence the result. Here, culture independent method is used. Further studies are necessary on the influence of humidity in order to get a clear picture.
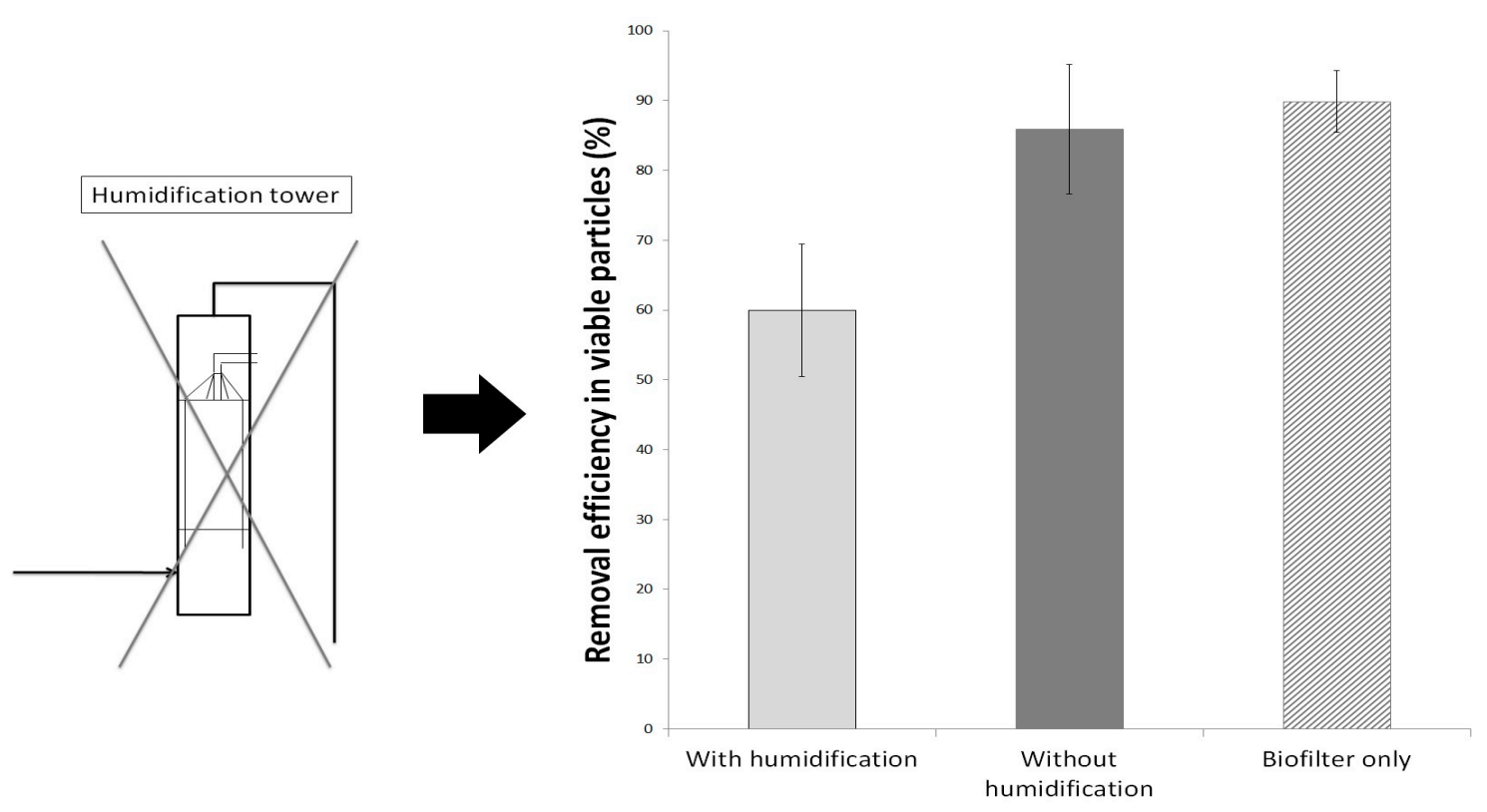

Figure 4. $R E_{\mathrm{vp}}$ during normal operation humidification and after $46 \mathrm{~h}$ without humidification. "With humidification" and "Without humidification" correspond to the evaluation of viable particles counts by using one and three sampling points. "Biofilter only" corresponds to the evaluation of viable particles counts by using sampling points 2 and $3(n=2)$.

This phenomenon could be explained as follows. The first assumption could be linked to the loss of cell viability when the air humidity ratio is reduced, thus resulting in a higher death rate among the microbial flora and the increase of the $\mathrm{RE}_{\mathrm{vp}}$.

However, the same concentration of viable particles was found at the fan aspiration and at the bottom of the biofilter when the air humidification was stopped, hence demonstrating a $\mathrm{RE}_{\mathrm{vp}}$ of $0 \%$ in the humidification tower. These results lead to think another explicative phenomenon is possible.

This second phenomenon could be due to the increase of the impaction phenomenon when the interstitial channels dry out (Figure 5). In normal water-saturated operation, the gaseous effluent travels through the packing bed material and passes near or through the interstitial water, creating turbulences, bubbles and agitation in the water. The impaction of micro-organisms into a collection liquid has been studied through the impingement technology, including the re-aerosolization mechanisms [20,44]. Even inside specifically designed collection devices, a consequent amount of liquid-impacted micro-organisms are re-aerosolized due to the effects of the airflow on the water. It is then possible that the interstitial water act as a collection liquid when the air passes near, with a consequent amount of micro-organisms re-aerosolized from it which then reached the outlet effluent. 
When the relative humidity of air is reduced, the passage of water-unsaturated air stream through a water saturated packing bed induces the volatilization of water molecules from interstitial water to restore liquid gas equilibrium. Consequently, the biofilm becomes thinner. The diffuse water phase diminishes, and the water level comes closer to the poly-saccharides structures of the biofilm [45-49]. The micro-organisms have easier access to the complex microscopic surface of the wooden chips. The re-aerosolization phenomenon is then reduced inducing the augmentation of the $\mathrm{RE}_{\mathrm{vp}}$.

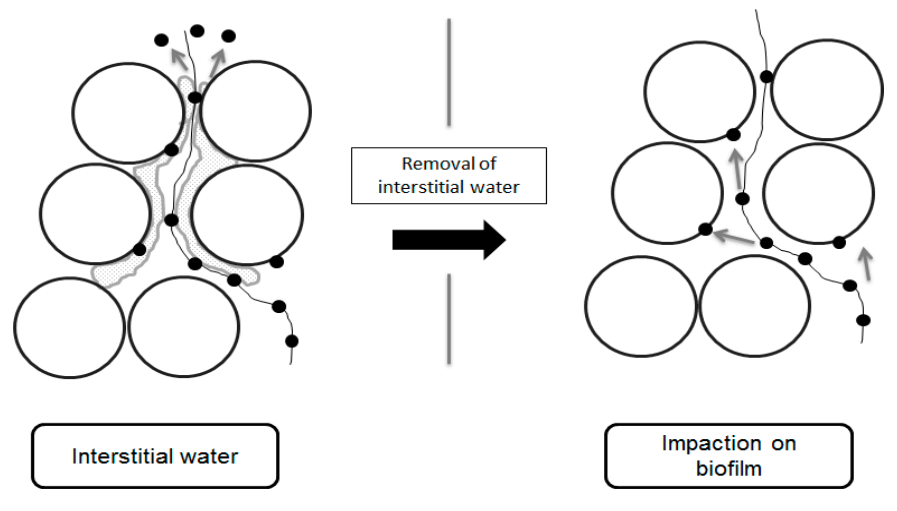

Figure 5. Explaining mechanism: disappearance of interstitial water and enhancement of the impaction phenomenon.

The relative humidity of both gaseous effluent and packing bed are then important parameters to ensure pollutant degradation and airborne viable particles removal capacities of biofilters.

Moreover, these results highlight the opposition between the best conditions for viable particles removal and for pollutant removal, as already highlighted by Fletcher et al. [6]. However, further tests are needed to confirm these results by exploring the composition of microbial communities notably using biomolecular tools.

\section{Conclusions}

This study brings new insight on viable particle emissions by biofilters. It has been highlighted that biofilters act as filters for viable micro-organisms. With stable conditions, a high and stable $\mathrm{RE}_{\mathrm{vp}}$ was maintained around $77 \%$ for both biofilters and enlightened the statistical equivalence between the two identical biofilters. Furthermore, this effect was linked to the biofilter itself and not to the humidification tower. The robustness of biofilters has been emphasized: a long pollutant deprivation period was required to largely reduce the viable particle $\mathrm{RE}_{\mathrm{vp}}$, thus showing the resistance and resilience of the microbial flora toward short term famine. Moreover, it has been stressed that interstitial water can negatively affect the $\mathrm{RE}_{\mathrm{vp}}$. Consequently, it was demonstrated that the operating parameters can influence the $\mathrm{RE}_{\mathrm{vp}}$.

Moreover, these results highlighted how the best operating conditions for airborne viable particles control are opposed to the best pollutant degradation conditions. This could indicate that if viable particles removal also becomes a focus, a compromise and balance should be reached in order to maximize both the pollutant and the $\mathrm{RE}_{\mathrm{vp}}$ but with the inability to reach the highest possible level for each.

Acknowledgments: We thank CMI Europe-Environment and IMT Mines Ales for funding this study as part of R. Soret Ph.D. program. This work was supported by the French Agency for Environmental and Energy Management (ADEME; grant agreement 1506C0053).

Author Contributions: Rémi Soret, Sandrine Bayle, Luc Malhautier, Philippe Geiger and Jean-Louis Fanlo conceived and designed the experiments; Rémi Soret performed the experiments; Rémi Soret, Sandrine Bayle, Luc Malhautier, Philippe Geiger and Jean-Louis Fanlo analyzed the data; Rémi Soret wrote the paper.

Conflicts of Interest: The authors declare no conflict of interest. 


\section{References}

1. Brinkmann, T.; Giner Santonja, G.; Yükseler, H.; Roudier, S.; Delgado Sancho, L. Best Available Techniques (BAT) Reference Document for Common Waste Water and Waste Gas Treatment/Management Systems in the Chemical Sector; EUR 28112; Publications Office of the European Union: Luxembourg, 2016; ISBN 9789279619960.

2. European IPPC Bureau. Reference Document on the Best Available Techniques for Waste Treatments Industries; European IPPC: Seville, Spain, 2006; p. 592.

3. Swanson, W.J.; Loehr, R.C. Biofiltration: Fundamentals, design and operations, and applications. J. Environ. Eng. 1997, 123, 538-546. [CrossRef]

4. Ottengraf, S.P.P.; Konings, J.H.G. Emission of microorganisms from biofilters. Bioprocess Eng. 1991, 7, 89-96. [CrossRef]

5. Sanchez-Monedero, M.A.; Stentiford, E.I.; Mondini, C. Biofiltration at composting facilities: Effectiveness for bioaerosol control. Environ. Sci. Technol. 2003, 37, 4299-4303. [CrossRef] [PubMed]

6. Fletcher, L.A.; Jones, N.; Warren, L.; Stentiford, E.I. Understanding Biofilter Performance and Determining Emission Concentrations under Operational Conditions; Final Report Project Number ER36; University of Leeds: Leeds, UK, 2014.

7. Pearson, C.; Littlewood, E.; Douglas, P.; Robertson, S.; Gant, T.W.; Hansell, A.L. Exposures and health outcomes in relation to bioaerosol emissions from composting facilities: A systematic review of occupational and community studies. J. Toxicol. Environ. Health 2015, 18, 43-69. [CrossRef] [PubMed]

8. British Government Bioaerosol Monitoring at Regulated Facilities-Use of M9: RPS 209-GOV.UK. Available online: https://www.gov.uk/government/publications/bioaerosol-monitoring-at-regulatedfacilities-use-of-\%0Am9-rps-209/bioaerosol-monitoring-at-regulated-facilities-use-of-m9-rps-209\%0A (accessed on 26 February 2018).

9. Esquivel-Gonzalez, S.; Aizpuru, A.; Patrón-Soberano, A.; Arriaga, S. Characterization of bioaerosol emissions from two biofilters during treatment of toluene vapours using epifluorescence microscopy. Int. Biodeterior. Biodegrad. 2017, 123, 78-86. [CrossRef]

10. Ghosh, B.; Lal, H.; Srivastava, A. Review of bioaerosols in indoor environment with special reference to sampling, analysis and control mechanisms. Environ. Int. 2015, 85, 254-272. [CrossRef] [PubMed]

11. Amann, R.I.; Ludwig, W.; Schleifer, K.H. Phylogenetic identification and in situ detection of individual microbial cells without cultivation. Microbiol. Rev. 1995, 59, 143-169. [CrossRef] [PubMed]

12. Tresse, O.; Lescob, S.; Rho, D. Dynamics of living and dead bacterial cells within a mixed-species biofilm during toluene degradation in a biotrickling filter. J. Appl. Microbiol. 2003, 94, 849-855. [CrossRef] [PubMed]

13. Khammar, N.; Malhautier, L.; Degrange, V.; Lensi, R.; Godon, J.J.; Fanlo, J.L. Link between spatial structure of microbial communities and degradation of a complex mixture of volatile organic compounds in peat biofilters. J. Appl. Microbiol. 2005, 98, 476-490. [CrossRef] [PubMed]

14. Yao, M.; Mainelis, G. Effect of physical and biological parameters on enumeration of bioaerosols by portable microbial impactors. J. Aerosol Sci. 2006, 37, 1467-1483. [CrossRef]

15. Yao, M.; Mainelis, G. Investigation of Cut-Off Sizes and Collection Efficiencies of Portable Microbial Samplers. Aerosol Sci. Technol. 2006, 40, 595-606. [CrossRef]

16. Yao, M.; Mainelis, G. Analysis of portable impactor performance for enumeration of viable bioaerosols. J. Occup. Environ. Hyg. 2007, 4, 514-524. [CrossRef] [PubMed]

17. Yao, M.; Mainelis, G. Use of portable microbial samplers for estimating inhalation exposure to viable biological agents. J. Expo. Sci. Environ. Epidemiol. 2007, 17, 31-38. [CrossRef] [PubMed]

18. Therkorn, J.H.; Mainelis, G. Effect of Agar Plate Volume on Accuracy of Culturable Bioaerosol Impactors. Aerosol Sci. Technol. 2013, 47, 1353-1362. [CrossRef]

19. Mainelis, G.; Tabayoyong, M. The Effect of Sampling Time on the Overall Performance of Portable Microbial Impactors. Aerosol Sci. Technol. 2010, 44, 75-82. [CrossRef]

20. Han, T.; Mainelis, G. Investigation of inherent and latent internal losses in liquid-based bioaerosol samplers. J. Aerosol Sci. 2012, 45, 58-68. [CrossRef]

21. Savage, N.; Krentz, C.; Könemann, T.; Han, T.T.; Mainelis, G.; Pöhlker, C.; Huffman, J.A. Systematic Characterization and Fluorescence Threshold Strategies for the Wideband Integrated Bioaerosol Sensor (WIBS) Using Size-Resolved Biological and Interfering Particles. Atmos. Meas. Tech. 2017, 10, 4279-4302. [CrossRef] 
22. Kirschman, D.; Lloyd, B. Ultraviolet In-Room Air Disinfection and Recirculation Greatly Reduces Viable Airborne Particle Concentrations in the Operating Room Setting; Aerobiotix, Inc.: West Carrollton, OH, USA, 2014.

23. United States Pharmacopeial Convention USP. 2015. <1223> Validation of Alternative Microbiological Methods; United States Pharmacopeial Convention: Rockville, MD, USA, 2015.

24. United States Pharmacopeial Convention USP. General Chapter <1116> Microbiological Control and Monitoring of Aseptic Processing Environments; United States Pharmacopeial Convention: Rockville, MD, USA, 2013; pp. 784-794.

25. European Pharmacopoeia 5.1.6. Alternative Methods for Control of Microbiological Quality. Eur. Pharmacopoeia 2006, 5.5, 4131-4142; European Directorate for the Quality of Medecines \& HealthCare: Strasbourg, France, 2006.

26. Parenteral Drug Association (PDA). Technical Report 33: Evaluation, Validation and Implementation of Alternative and Rapid Microbiological Methods. J. Pharm. Sci. Technol. 2013, 54, 1-39.

27. TSI BioTrak ${ }^{\circledR}$ Real-Time Viable Particle Counter Sample and Collection Efficiency; Application Note CC-104; TSI Incorporated: Shoreview, MN, USA, 2014; pp. 1-7.

28. Li, M.; Qi, J.; Zhang, H.; Huang, S.; Li, L.; Gao, D. Concentration and size distribution of bioaerosols in an outdoor environment in the Qingdao coastal region. Sci. Total Environ. 2011, 409, 3812-3819. [CrossRef] [PubMed]

29. Seedorf, J.; Hartung, J. Reduction efficiencies of a biofilter and a bio-scrubber as bio-aerosols in two piggeries. Berl. Munch. Tierarztl. Wochenschr. 1999, 112, 444-447. [PubMed]

30. Martens, W.; Martinec, M.; Zapirain, R.; Stark, M.; Hartung, E.; Palmgren, U. Reduction potential of microbial, odour and ammonia emissions from a pig facility by biofilters. Int. J. Hyg. Environ. Health 2001, 203, 335-345. [CrossRef] [PubMed]

31. Seedorf, J.; Hartung, J. Reduction efficiency of a container-based biofilter for bioaerosols from a broiler house. Anim. Prod. Aust. 2002, 24, 205-208.

32. Schlegelmilch, M.; Herold, T.; Streese, J.; Hensel, A.; Stegmann, R. The potential to reduce emissions of airborne microorganisms by means of biological waste gas treatment systems. Waste Manag. 2005, 25, 955-964. [CrossRef] [PubMed]

33. Haumacher, R.; Philipp, W.; Böhm, R. Bioaerosols from composting-quantitative measurements on biofilters and non-thermal plasma technology. ISAH 2005, 2, 276-278.

34. Chmielowiec-Korzeniowska, A.; Tymczyna, L.; Skórska, C.; Sitkowska, J.; Cholewa, G.; Dutkiewicz, J. Efficacy of a novel biofilter in hatchery sanitation: I. Removal of airborne bacteria, dust and endotoxin. Ann. Agric. Environ. Med. 2007, 14, 141-150. [CrossRef]

35. Ho, K.L.; Chung, Y.C.; Lin, Y.H.; Tseng, C.P. Microbial populations analysis and field application of biofilter for the removal of volatile-sulfur compounds from swine wastewater treatment system. J. Hazard. Mater. 2008, 152, 580-588. [CrossRef] [PubMed]

36. Zhao, Y.; Aarnink, A.J.A.; De Jong, M.C.M.; Ogink, N.W.M.; Koerkamp, P.G. Effectiveness of multi-stage scrubbers in reducing emissions of air pollutants from pig houses. Trans. ASABE 2011, 54, 285-293. [CrossRef]

37. Tymczyna, L.; Chmielowiec-Korzeniowska, A.; Raczyńska, J.; Drabik, A. Removal of microbial contaminants from pig house air using biofilter organic media. Ann. Anim. Sci. 2011, 11, 453-464.

38. Song, J.H.; Kinney, K.A. Effect of vapor-phase bioreactor operation on biomass accumulation, distribution, and activity: Linking biofilm properties to bioreactor performance. Biotechnol. Bioeng. 2000, 68, 508-516. [CrossRef]

39. Irvine, R.L.; Moe, W.M. Period biofilter operation for enhanced performance during unsteady-state loading conditions. Water Sci. Technol. 2001, 43, 231-239. [PubMed]

40. Wright, W.F. Transient response of vapor-phase biofilters. Chem. Eng. J. 2005, 113, 161-173. [CrossRef]

41. Cabrol, L. Evaluation de le Robustesse dun Système de Biofiltration d'Effluent de Compostage. Ph.D. Thesis, University of Montpellier II, Montpellier, France, 2010.

42. Cabrol, L.; Malhautier, L. Integrating microbial ecology in bioprocess understanding: The case of gas biofiltration. Appl. Microbiol. Biotechnol. 2011, 90, 837-849. [CrossRef] [PubMed]

43. Vergara-Fernández, A.; Salgado-Ísmodes, V.; Pino, M.; Hernández, S.; Revah, S. Temperature and moisture effect on spore emission in the fungal biofiltration of hydrophobic VOCs. J. Environ. Sci. Health Part A 2012, 47, 605-613. [CrossRef] [PubMed] 
44. Grinshpun, S.A.; Willeke, K.; Ulevicius, V.; Juozaitis, A.; Terzieva, S.; Donnelly, J.; Stelma, G.N.; Brenner, K.P. Effect of impaction, bounce and reaerosolization on the collection efficiency of impingers. Aerosol Sci. Technol. 1997, 26, 326-342. [CrossRef]

45. Costerton, J.W.; Geesey, G.G.; Cheng, K.J. How bacteria stick. Sci. Am. 1978, 238, 86-95. [CrossRef] [PubMed]

46. Costerton, J.W.; Lewandowski, Z.; DeBeer, D.; Caldwell, D.; Korber, D.; James, G. Biofilms, the customized microniche. J. Bacteriol. 1994, 176, 2137-2142. [CrossRef] [PubMed]

47. Watnick, P.; Kolter, R. Biofilm, city of microbes. J. Bacteriol. 2000, 182, 2675-2679. [CrossRef] [PubMed]

48. Heydorn, A.; Heydorn, A.; Nielsen, A.T.; Nielsen, A.T.; Hentzer, M.; Hentzer, M. Quantication of biofilm structures by the novel computer program. Image Process. 2000, 2395-2407. [CrossRef]

49. Bridier, A.; Le Coq, D.; Dubois-Brissonnet, F.; Thomas, V.; Aymerich, S.; Briandet, R. The spatial architecture of Bacillus subtilis biofilms deciphered using a surface-associated model and in situ imaging. PLoS ONE 2011, 6, e16177. [CrossRef] [PubMed]

(C) 2018 by the authors. Licensee MDPI, Basel, Switzerland. This article is an open access article distributed under the terms and conditions of the Creative Commons Attribution (CC BY) license (http://creativecommons.org/licenses/by/4.0/). 